Pacific Journal of Mathematics

AN ESTIMATE OF THE NIELSEN NUMBER AND AN
EXAMPLE CONCERNING THE LEFSCHETZ FIXED POINT
THEOREM

DAN MCCORD 


\title{
AN ESTIMATE OF THE NIELSEN NUMBER AND AN EXAMPLE CONCERNING THE LEFSCHETZ FIXED POINT THEOREM
}

\section{DAN MCCORD}

\begin{abstract}
Given a map $f: X \rightarrow X$ of a compact ANR and any finite connected regular covering $p: \tilde{X} \rightarrow X$ to which $f$ admits lifts, then one can compute a certain homotopy invariant $N_{H}(f)$ if the Lefschetz numbers of the lifts and the relation of the lifts to the covering transformations are known. $H=p_{*} \pi_{1}(\tilde{X})$. Every map homotopic to $f$ has at least $N_{H}(f)$ fixed points. If $X$ is a finite polyhedron, then $N_{H}(f) \leqq N(f)$, the Nielsen number. The smaller invariant is easier to compute by virtue of its smallness, but it is adequate to discern for example homeomorphisms, $h$, of manifolds in all dimensions with $L(h)=0$ and $N(h) \geqq 2$.
\end{abstract}

1. Introduction. It is known that if $X$ is simply-connected and either a compact topological manifold [2] or a finite polyhedron satisfying the Shi condition [1, p. 139], then the converse of the Lefschetz Fixed Point Theorem is valid, i.e. if the Lefschetz number $L(f)$ of a map $f: X \rightarrow X$ is zero, then there is a map $g: X \rightarrow X$ homotopic to $f$ which has no fixed points. This converse remains valid if the condition of simple-connectivity is relaxed to that of Jiang [1, p. 141].

Our objective here is to give examples of manifolds $M^{n}$ in all dimensions which admit self-maps $f$ (homeomorphisms, in fact) with $L(f)=0$ such that every map homotopic to $f$ has two or more fixed points.

We will use an approach due to G. Hirsch [3] which detects essential Nielsen classes using two-fold covers. In the following section we outline a generalization of this procedure.

2. The generalized Hirsch method. Let $X$ be a compact ANR and $p: \tilde{X} \rightarrow X$ a finite connected regular covering of $X$. Let $H=p_{\#} \pi_{1}(\tilde{X})$. For maps $f: X \rightarrow X$ which admit lifts $\tilde{f}$,

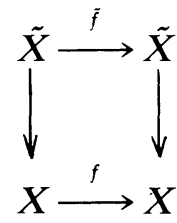

we will define a number $N_{H}(f)$ which is no larger than the Nielsen 
number $N(f)$ and which is easier to compute because it may be smaller and because it is defined with reference to $\tilde{f}_{*}: H_{*}(\tilde{X}) \rightarrow H_{*}(\tilde{X})$ rather than to the local fixed point index.

Let $f$ be as mentioned, and notice that since $p$ is regular, we have assumed there is a collection

$$
\mathscr{C}=\{\tilde{f} \mid p \tilde{f}=f p\}
$$

of lifts having as many members as the multiplicity of $p$. Let Fix ( $g$ ) denote the set of points fixed by a map $g$.

If $\tilde{f} \in \mathscr{C}$, then

$$
p \operatorname{Fix}(\tilde{f}) \subset \operatorname{Fix}(f)
$$

If $\tilde{f}, \tilde{f}^{\prime} \in \mathscr{C}$ and

$$
p \operatorname{Fix}(\tilde{f}) \cap p \operatorname{Fix}\left(\tilde{f}^{\prime}\right) \neq \phi,
$$

then there is a covering transformation $\gamma: \tilde{X} \rightarrow \tilde{X}$ such that

$$
\tilde{f}^{\prime} \gamma=\gamma \tilde{f}
$$

Whenever the conjugacy relation (1) prevails, we find that

$$
p \operatorname{Fix}(\tilde{f})=p \operatorname{Fix}\left(\tilde{f}^{\prime}\right)
$$

It is convenient to summarize this situation in the following way. The group $G$ of covering transformations acts on $\mathscr{C}$ by conjugation, partitioning $\mathscr{C}$ into a collection $\mathscr{C} / G$ of (let us say $k$ ) conjugacy classes.

To each class $[\tilde{f}] \in \mathscr{C} / G$ we may associate the subset

$$
p \operatorname{Fix}(\tilde{f}) \subset \operatorname{Fix}(f)
$$

independently of the representative $\tilde{f}$. These various subsets of Fix $(f)$ are mutually disjoint; and moreover,

$$
\operatorname{Fix}(f)=\bigcup_{[\tilde{f}] \in \mathscr{G} / G} p \operatorname{Fix}(\tilde{f})
$$

Likewise, to each class $[\tilde{f}] \in \mathscr{C} / G$ we may associate the number

$$
L([\tilde{f}])=L(\tilde{f})
$$


independently of the representative $\tilde{f}$. These numbers constitute an unordered $k$-tuple $\mathscr{L}_{H}(f)$.

THEOREM 1. $\mathscr{L}_{H}(f)$ is a homotopy invariant.

Proof. Let $F: X \times I \rightarrow X$ be a homotopy with

$$
F(x, 0)=f_{0}(x), \quad F(x, 1)=f_{1}(x) .
$$

For $i=0,1$, let

$$
\mathscr{C}_{\imath}=\left\{\tilde{f}_{\imath} \mid p \tilde{f}_{\imath}=f_{i} p\right\}
$$

be the collection of lifts of $f_{i}$.

For each lift $\tilde{f}_{0} \in \mathscr{C}_{0}$ of $f_{0}$ there is a unique homotopy $\tilde{F}$ completing the diagram

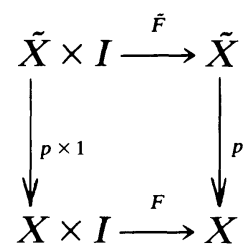

and satisfying the initial condition

$$
\tilde{F}(x, 0)=\tilde{f}_{0}(x) .
$$

By associating with $\tilde{f}_{0}$ the other end of this homotopy

$$
\tilde{f}_{1}(x)=\tilde{F}(x, 1)
$$

we may define a one-to-one correspondence

$$
\eta: \mathscr{C}_{0} \rightarrow \mathscr{C}_{1}
$$

Corresponding lifts have the same Lefschetz number, and a conjugate pair of lifts in $\mathscr{C}_{0}$ correspond with a pair in $\mathscr{C}_{1}$ which are also conjugate. This completes the proof of Theorem 1 .

Definition. Let $N_{H}(f)$ be the number of classes $[\tilde{f}] \in \mathscr{C} / G$ for which $L(\tilde{f}) \neq 0$.

THEOREM 2. Every map homotopic to $f$ has at least $N_{H}(f)$ fixed points. 
Proof. By Theorem 1, we need only consider $f$ itself. Certainly $p$ Fix $(\tilde{f}) \neq \phi$ whenever $L(\tilde{f}) \neq 0$, and these subsets of Fix $(f)$ are mutually disjoint if they derive from different conjugacy classes.

Corollary 1 (Hirsch). Let $f: X \rightarrow X$ be a map of a compact ANR and $p: \tilde{X} \rightarrow X$ a connected two-fold covering of $X$. Suppose

(1) f lifts to $\tilde{f}, \tilde{f}^{\prime}: \tilde{X} \rightarrow \tilde{X}$,

(2) if $p(a)=p(b)$ and $a \neq b$, then $\tilde{f}(a) \neq \tilde{f}(b)$,

(3) $L(\tilde{f}) \neq 0 \neq L\left(\tilde{f}^{\prime}\right)$.

Then every map homotopic to $f$ has two or more fixed points.

Proof. If $\gamma$ is the nontrivial covering transformation, then by (2), $\gamma \tilde{f}=\tilde{f} \gamma ;[\tilde{f}] \neq\left[\tilde{f}^{\prime}\right]$.

3. Relation of the Hirsch method to the Nielsen number. Suppose $X, \tilde{X}, p, f$ are as above. Let us retain the notation used before, and let $N(f)$ denote the Nielsen number of $f$. Later in this section, we will prove the following.

THEOREM 3. If $X$ is a finite polyhedron, then

$$
N(f) \geqq N_{H}(f) .
$$

Recall [1] that $N(f)$ is the number of fixed point (equivalence) classes $F \subset$ Fix $(f)$ for which the local fixed point index $i(F) \neq 0$. Here the equivalence relation is this: $x_{0}, x_{1} \in \operatorname{Fix}(f)$ are equivalent if there is a path $\omega: I \rightarrow X$ with $\omega(0)=x_{0}, \omega(1)=x_{1}$, and

$$
\left[\omega(f \omega)^{-1}\right]=1 \in \pi_{1}(X) .
$$

The other invariant, $N_{H}(f)$, is also related to an equivalence relation on Fix $(f)$ as defined by the partition (2).

LEMma 1. For $x_{0}, x_{1} \in \operatorname{Fix}(f)$, the following are equivalent:

(1) $x_{0}, x_{1} \in p \operatorname{Fix}(\tilde{f})$ for some $\tilde{f} \in \mathscr{C}$

(2) there is a path $\omega: I \rightarrow X$ with $\omega(0)=x_{0}, \omega(1)=x_{1}$, and

$$
\left[\omega(f \omega)^{-1}\right] \in H=p_{\#} \pi_{1}(\tilde{X}) .
$$

Proof. Supposing (1), choose $\tilde{x}_{i} \in p^{-1}\left(x_{i}\right) \cap \operatorname{Fix}(\tilde{f})$, for $i=0$ and 1, and next choose a path $\tilde{\omega}: I \rightarrow \tilde{X}$ with $\tilde{\omega}(0)=\tilde{x}_{0}, \tilde{\omega}(1)=\tilde{x}_{1}$. Then $\omega=p \tilde{\omega}$ satisfies (2). 
Supposing (2), choose any $\tilde{x}_{0} \in p^{-1}\left(x_{0}\right)$ and then choose $\tilde{f} \in \mathscr{C}$ with $\tilde{f}\left(\tilde{x}_{0}\right)=\tilde{x}_{0}$. The paths $\omega$ and $f \omega$ lift to paths starting at $\tilde{x}_{0}$ and ending at a common point $\tilde{x}_{1}=\tilde{f}\left(\tilde{x}_{1}\right) \in p^{-1}\left(x_{1}\right)$. So $x_{0}, x_{1} \in p \operatorname{Fix}(\tilde{f})$.

A result of this first Lemma is that each Nielsen class $F$ has the property

$$
F \subset p \operatorname{Fix}(\tilde{f})
$$

for some $[\tilde{f}] \in \mathscr{C} / G$. And so each of the sets $p \operatorname{Fix}(\tilde{f})$ is the union of several Nielsen classes.

Definition. For $[\tilde{f}] \in \mathscr{C} / G$, let $m([\tilde{f}])$ be the number of covering transformations $\gamma \in G$ for which $\tilde{f}=\gamma \tilde{f} \gamma^{-1}$. That is,

$$
m([\tilde{f}])=\operatorname{order}(\text { stabilizer of } \tilde{f} \text { in } G) .
$$

Lemma 2. If $\tilde{f} \in \mathscr{C}$ and $x \in p \operatorname{Fix}(\tilde{f})$, then

$$
m([\tilde{f}])=\operatorname{cardinality}\left(p^{-1}(x) \cap \operatorname{Fix}(\tilde{f})\right) .
$$

Proof. Where $\tilde{x} \in p^{-1}(x) \cap \operatorname{Fix}(\tilde{f})$, it is easily shown that $\gamma \tilde{x} \in$ $\operatorname{Fix}(\tilde{f})$ if and only if $\tilde{f} \gamma=\gamma \tilde{f}$.

Lemma 3. If $\operatorname{Fix}(f)$ is finite and $\tilde{f} \in \mathscr{C}$, then

$$
L(\tilde{f})=m([\tilde{f}]) \cdot i(p \operatorname{Fix}(\tilde{f}))
$$

where $i(p \operatorname{Fix}(\tilde{f}))$ is the local fixed point index of $f$ in a closed neighborhood of $p \operatorname{Fix}(\tilde{f})$ disjoint with the remainder of $\operatorname{Fix}(f)$.

Proof. Since $p$ is locally a homeomorphism, the index of a fixed point $x \in p \operatorname{Fix}(\tilde{f})$ is the same as that of each of the $m([\tilde{f}])$ points in $p^{-1}(x) \cap \operatorname{Fix}(\tilde{f})$.

Proof of Theorem 3. Since both $N(f)$ and $N_{H}(f)$ are homotopy invariant, we may assume that $\operatorname{Fix}(f)$ is finite (Hopf construction for finite polyhedra, [1]). By Lemma 3, $L(\tilde{f})$ is a nonzero multiple of the sum of the indices $i(F), F \subset p \operatorname{Fix}(\tilde{f})$. Thus if $L(\tilde{f}) \neq 0$, then $i(F) \neq 0$ for at least one of the fixed point classes $F \subset p \operatorname{Fix}(\tilde{f})$.

REMARK. If $p: \tilde{X} \rightarrow X$ is the universal covering space $\left(\pi_{1}(X)\right.$ finite and $H=\{1\}$ ), then the nonempty sets $p$ Fix $(\tilde{f})$ are precisely the Nielsen fixed point classes, and $N(f)=N_{H}(f)$. This is the case covered by Jiang [4]. 
4. An example in dimension two. We will exhibit a homeomorphism $f: X \rightarrow X$ of the orientable surface of genus 2 for which $L(f)=0$ and to which Corollary 1 applies. We will employ certain elementary surface homeomorphisms that have been described by W. B. R. Lickorish [5]:

Let the 2-manifold $M$ contain an annulus, $A$, one of the boundary components of which is a simple closed curve $c$. There is a homeomorphism of $A$ to itself, fixed on the boundary of $A$, which sends radial arcs onto arcs which spiral once [or several times] around $A$ (see Figure 1). This can be extended to a homeomorphism of $M$ to itself, by the identity on $M-A$. Intuitively this homeomorphism can be thought of as the process of cutting $M$ along $c$, twisting one of the now free ends, and then gluing together again.

Our double covering of $X$ is by $\tilde{X}$, the orientable surface of genus 3. As indicated in Figure 2, such a covering is obtained by wrapping the center hole of $\tilde{X}$ twice around the left hole of $X$. Alternatively, this projection may be regarded as the process of cutting $\tilde{X}$ along the two unlabeled simple closed curves and then mapping each half onto $X$ by first identifying its two boundary components and then mapping the resulting space homeomorphically onto $X$ so that the identified curve maps onto the unlabeled simple closed curve in $X$. The other curves in Figure 2 are the free Abelian generators of $H_{1}(X)$ and $H_{1}(\tilde{X})$.

The homeomorphism $f: X \rightarrow X$ is a composition of Lickorish twists. On the left hole of $X$ first perform a single twist at $\beta_{1}$ twisting in the direction that sweeps $\alpha_{1}$ backward along $\beta_{1}$, and then perform a double twist at $\alpha_{1}$ in the direction that sweeps $\beta_{1}$ forward along $\alpha_{1}$. The effect of this composition on the two generators $\alpha_{1}, \beta_{1}$ is described by the matrix

$$
\left(\begin{array}{rr}
1 & -1 \\
0 & 1
\end{array}\right)\left(\begin{array}{ll}
1 & 0 \\
2 & 1
\end{array}\right)=\left(\begin{array}{rr}
-1 & -1 \\
2 & 1
\end{array}\right)
$$

Do nothing to the other hole of $X$ so that $\alpha_{2}, \beta_{2}$ are transformed by the identity matrix. Since $f$ is an orientation preserving homeomorphism,

$$
f_{*}=i d: H_{2}(X) \rightarrow H_{2}(X) .
$$

And thus $L(f)=1-2+1=0$.

The lifts $\tilde{f}, \tilde{f}^{\prime}$ of $f$ are each a composition of twists (and covering transformations). We may describe the more obvious one, $\tilde{f}$, as follows. On the center hole, $\tilde{f}$ is two twists at $\tilde{\beta}_{2}$ in the direction that sweeps $\tilde{\alpha}_{2}$ backward along $\tilde{\beta}_{2}$ followed by a single twist at $\tilde{\alpha}_{2}$ in the direction that sweeps $\tilde{\beta}_{2}$ forward along $\tilde{\alpha}_{2}$. The associated matrix is 

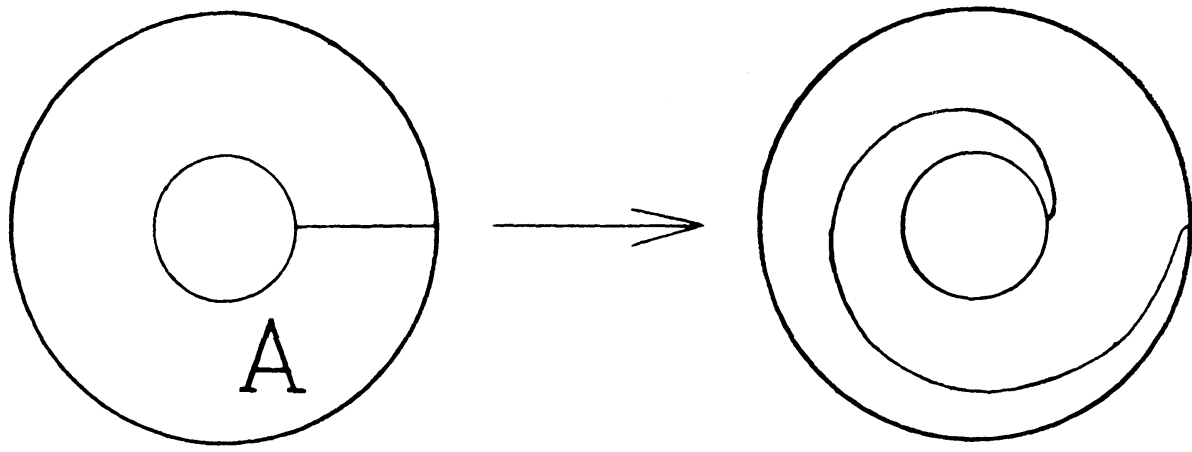

Figure 1

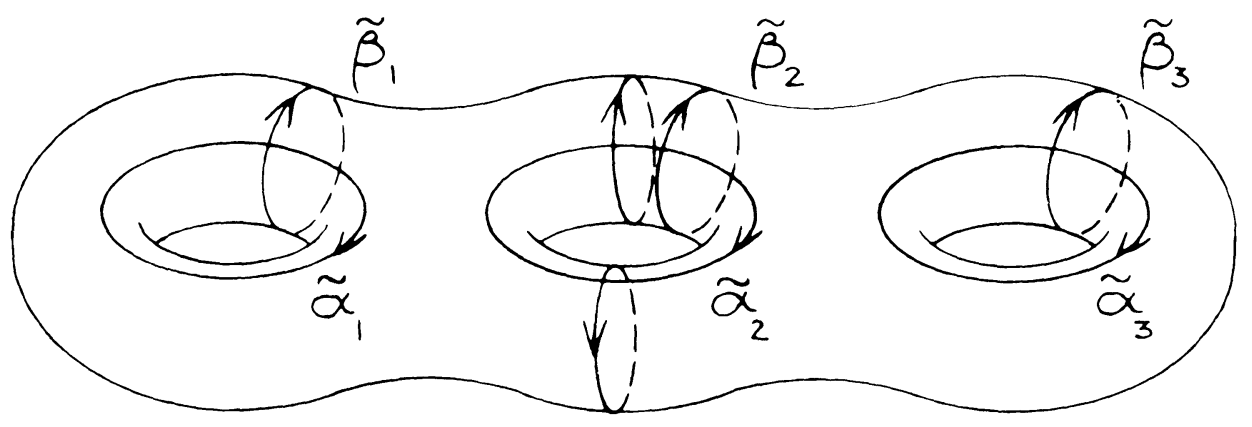

$\sqrt{1}$

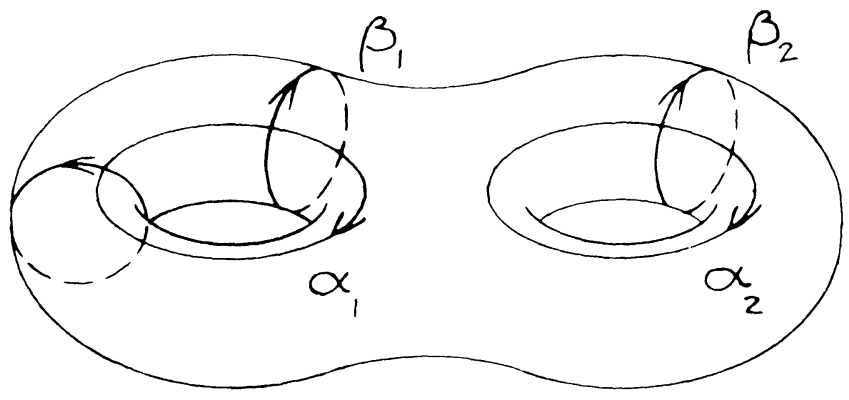

Figure 2 


$$
\left(\begin{array}{rr}
1 & -2 \\
0 & 1
\end{array}\right)\left(\begin{array}{ll}
1 & 0 \\
1 & 1
\end{array}\right)=\left(\begin{array}{rr}
-1 & -2 \\
1 & 1
\end{array}\right)
$$

The other holes are undisturbed. We find $L(\tilde{f})=1-4+1=-2$.

Since $\tilde{f}_{*}^{\prime}=(\gamma \tilde{f})_{*}=\gamma_{*} \tilde{f}_{*}$, it is easily shown that $L\left(\tilde{f}^{\prime}\right)=+2$. Thus all of the conditions of Corollary 1 are satisfied.

5. Other examples. Let $f, X, p, \tilde{X}, \tilde{f}, \tilde{f}^{\prime}$ be as in the previous section. For $n \geqq 3$, let

$$
M^{n}=X \times S^{n-2}
$$

Let $g: S^{n-2} \rightarrow S^{n-2}$ be a homeomorphism for which $L(g) \neq 0$. And let us consider the homeomorphism

$$
h=f \times g: M^{n} \rightarrow M^{n} .
$$

There is the double covering

$$
p \times 1: \tilde{X} \times S^{n-2} \rightarrow X \times S^{n-2},
$$

and $h$ lifts to the homeomorphisms

$$
\tilde{f} \times g, \tilde{f}^{\prime} \times g: \tilde{X} \times S^{n-2} \rightarrow \tilde{X} \times S^{n-2} .
$$

Also

$$
L(h)=L(f) \cdot L(g)=0
$$

and

$$
L(\tilde{f} \times g)=L(\tilde{f}) \cdot L(g) \neq 0 \neq L\left(\tilde{f}^{\prime}\right) \cdot L(g)=L\left(\tilde{f}^{\prime} \times g\right) .
$$

So we may again conclude that although $L(h)=0, N(h) \geqq 2$.

\section{REFERENCES}

1. R. F. Brown, The Lefschetz Fixed Point Theorem; Scott, Foresman and Company, Glenview, Il., 1971.

2 E. Fadell, On a coincidence theorem of F. B. Fuller, Pacific J. Math., 15 (1965), 825-834.

3. G. Hirsch, Detérmination d'un nombre minimum de points fixes pour certaines représentations, Bull. Sci. Math., 64 (1940), 45-55. 
4. Jiang Bo-Ju, Estimation of the Nielsen numbers, Acta. Math. Sinica, 14 (1964), 304-312= Chinese Math. Acta., 5 (1964), 330-339.

5. W. B. R. Lickorish, Homeomorphisms of non-orientable two-manifolds, Proc. Camb. Phil. Soc., 59 (1963), 307-317.

6. D. McCord, The Converse of the Lefschetz fixed point theorem for surfaces and higher dimensional manifolds, doctoral dissertation, U. of Wis., 1970.

Received February 18, 1976 and in revised form May 26, 1976.

UNIVERSITY OF WISCONSIN - MADISON 




\section{Pacific Journal of Mathematics}

\section{Vol. 66, No. $1 \quad$ November, 1976}

Helen Elizabeth. Adams, Factorization-prime ideals in integral domains ............ Patrick Robert Ahern and Robert Bruce Schneider, The boundary behavior of Henkin's kernel.

Daniel D. Anderson, Jacob R. Matijevic and Warren Douglas Nichols, The Krull

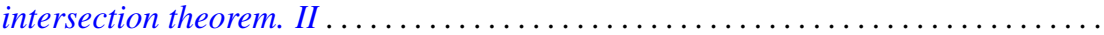

Efraim Pacillas Armendariz, On semiprime P.I.-algebras over commutative regular

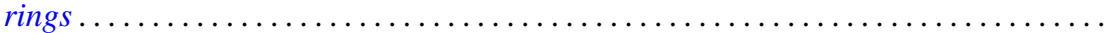

Robert H. Bird and Charles John Parry, Integral bases for bicyclic biquadratic fields

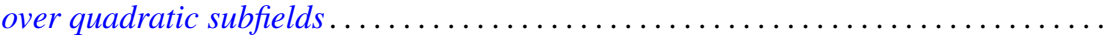

Tae Ho Choe and Young Hee Hong, Extensions of completely regular ordered

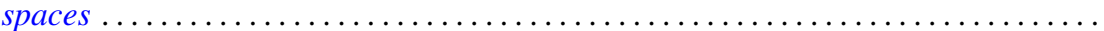

John Dauns, Generalized monoform and quasi injective modules ...............

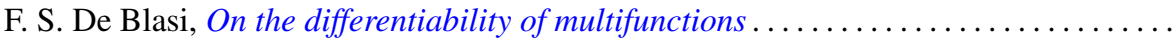

Paul M. Eakin, Jr. and Avinash Madhav Sathaye, R-endomorphisms of $R[[X]]$ are

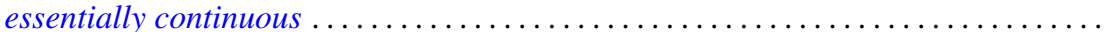

Larry Quin Eifler, Open mapping theorems for probability measures on metric

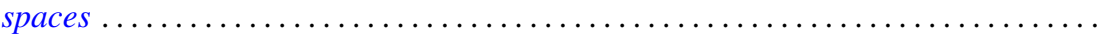

Garret J. Etgen and James Pawlowski, Oscillation criteria for second order self adjoint

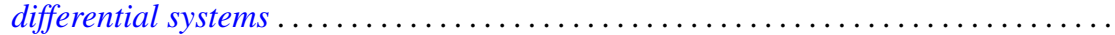

Ronald Fintushel, Local $S^{1}$ actions on 3-manifolds .

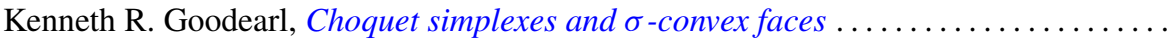

John R. Graef, Some nonoscillation criteria for higher order nonlinear differential

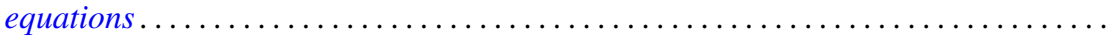

Charles Henry Heiberg, Norms of powers of absolutely convergent Fourier series: an example.

Les Andrew Karlovitz, Existence of fixed points of nonexpansive mappings in a space

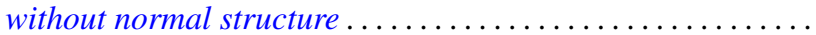

Gangaram S. Ladde, Systems of functional differential inequalities and functional

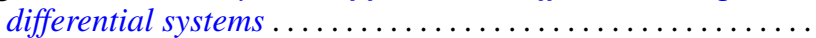

Joseph Michael Lambert, Conditions for simultaneous approximation and interpolation with norm preservation in $C[a, b]$.

Ernest Paul Lane, Insertion of a continuous function.

Robert F. Lax, Weierstrass points of products of Riemann surfaces .

Dan McCord, An estimate of the Nielsen number and an example concerning the Lefschetz fixed point theorem...

Paul Milnes and John Sydney Pym, Counterexample in the theory of continuous functions on topological groups...

Peter Johanna I. M. De Paepe, Homomorphism spaces of algebras of holomorphic functions

Judith Ann Palagallo, A representation of additive functionals on $L^{p}$-spaces,

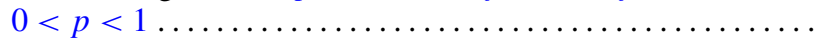

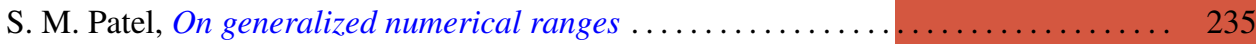

Thomas Thornton Read, A limit-point criterion for expressions with oscillatory

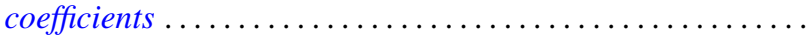

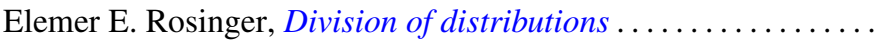

Peter S. Shoenfeld, Highly proximal and generalized almost finite

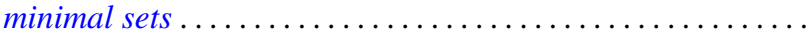

R. Sirois-Dumais and Stephen Willard, Quotient-universal sequential spaces

Robert Charles Thompson, Convex and concave functions of singular values of matrix sums....

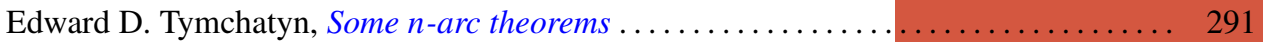

